\title{
COMBINING CONFOCAL AND BSE SEM IMAGING FOR BONE BLOCK SURFACES
}

\author{
Alan Boyde ${ }^{1,2}$, Ludek Lovicar ${ }^{1,3}$ and Jan Zamecnik Za $^{1,3}$ \\ ${ }^{1}$ Anatomy Dept., University College London, UK \\ ${ }^{2}$ Centre for Oral Growth and Development, Barts and The London School of Medicine and Dentistry, Queen Mary, \\ University of London, Turner St., London, UK \\ ${ }^{3}$ Faculty of Mechanical Engineering, Brno University of Technology, Brno, Czech Republic
}

\begin{abstract}
The present report presents a method for the correlation of qualitative and quantitative BSE SEM imaging with confocal scanning light microscopy (CSLM) imaging modes applied to bone samples embedded in PMMA. The SEM has a proper digital scan generator: we leave the BSE image unchanged, and match the CSLM image to it, because the CSLM scan mechanism is not digital, though the signal is digitised. Our overlapping program uses a linear transformation matrix which projects one system to the other, calculated by finding three corresponding points in BSE and CSLM pictures.

BSE images are empty where cells and osteoid are present. Fluorescence mode CSLM fills in these gaps. The combination images enhance our understanding of what is going on - and re-establish the need for good cellular preservation.
\end{abstract}

Key Words Bone, scanning electron microscopy, confocal optical microscopy, block face microscopy, osteoid, osteoblasts, osteocytes

Address for correspondence:

Alan Boyde

Biophysics Section,

Centre for Oral Growth and Development,

Dental Institute,

Queen Mary,

University of London

New Road.,

London E1 1BB, UK

E-mail: a.boyde@qmul.ac.uk

\section{Introduction}

Due to structural damage in cutting, physical sectioning with a knife is not the best method for preparing calcified tissues for microscopical study if we wish to see cells and hard matrices in undisturbed relationships. We find that the study of tissue remaining in a block face, discarding the damaged tissue fragments as polishing or micromilling swarf, is the best basic approach. We therefore need to concern ourselves with means of cutting back close to the layer(s) to be studied whilst creating minimal disturbance to the remaining tissue, and choosing and optimising microscopic observation modes. No single means of study can give us all the information we require. No two means will give exactly the same class of information, and in most cases there will be significant differences in the depths and volumes interrogated by different methods. Thus we need to consider available modes and the best means of correlating them. In some cases, we will find it valuable to match information deriving from substantial depths, for example, the entire thickness of a 'block' which is a 100 micron thick section, with that stemming from the superficial micron, as in matching circularly polarised light data for collagen orientation with qBSE for mineral content (Goldman et al., 2000).

Compositional (atomic number contrast) mode BSE SEM of plastic embedded skeletal and dental tissues is of key value in demonstrating differences in the degree of mineralisation of hard tissue matrices at sub-micron resolution (Boyde and Jones, 1983; Roschger et al., 1995; Vajda et al., 1998; Boyde et al., 1999). Sample preparation technique is critical for the success of this approach, which assumes and requires that superficial topographic relief is minimal. Typically, diamond micro-milling of plastic embedded block faces will give a relief height of less than one-tenth of a micron, which is satisfactory. Polishing leads to the development of relief related to the underlying changes in collagen orientation in bone lamellae, due to related changes in microhardness and polishing wear resistance (Howell and Boyde, 1999).

Confocal scanning light microscopy (CSLM) revolutionised block face microscopy in hard tissues. Excellent structural information is obtained in the reflection-backscattering mode, albeit that such contrast is minimised in plastic embedded tissue and that most current commercial CSLMs function badly in this mode due to poor design in eliminating light reflected from optical component surfaces normal to the optic axis. In the fluorescence mode, however, they excel. There is usually sufficient auto-fluorescence signal to read general 
histology and identify and map cell types and matrix structure. This is more so in formaldehyde fixed tissue, and may be dazzling in glutaraldehyde preserved material. If this is not enough, a general fluorescence is created by staining with brilliant sulphaflavine in ethanolic solution (Boyde et al., 1990): this dye is stable to electron beam irradiation and can also be used for cathodoluminescence (CL) mode imaging in the SEM (Boyde and Reid, 1984). Several strongly fluorescent dyes have the remarkable property of binding most strongly within calcified tissue matrix which is calcifying at the time of intra-vital administration (Frost, 1962; Hulth and Olerud, 1962; Rahn and Perren, 1970, 1971), although their distribution at lower concentrations is widespread in all hydrophobic compartments (Boyde et al., 1995). These substances include alizarin, calceins, xylenol orange and the tetracycline antibiotics. Such growth- (mineralisation-) front labels are particularly well studied by fluorescence CSLM, where they can be mapped in 3D to 100 microns deep into a well cleared block face (Boyde, 1987). CL SEM also permits their study in the surface few microns (Boyde and Reid, 1983).

The present studies consider practical approaches to correlating qualitative and quantitative BSE SEM imaging with confocal imaging modes. We have applied the method in several problem areas, especially in studies of changes in response to exercise in articular calcified cartilage and subchondral bone of the equine third metacarpal bone, and bone changes in nephrectomised rats, such as here illustrated with examples from a study of human iliac crest bone biopsies from children presenting with deformed lower limbs (Schnitzler et al., 1994).

\section{Materials and Methods}

Bone samples (from the study of Schnitzler et al., 1994) were dehydrated in ethanol and embedded in poly-methylmethacrylate (PMMA). The block surfaces were finished by diamond ultra-milling (Reichert-Jung Polycut E).

For CSLM, a Leica model 2 with 10/0.40, 25/0.75, and $40 / 1.0$ oil immersion lenses was used, coupling a coverslip to the micromilled block surface using glycerine (which is easily removed by washing with distilled water). Particular care has to be used in levelling the sample prior to confocal imaging.

For SEM, we used a Zeiss DSM962 automated digital SEM with Kontron external control computer, operated mostly at $20 \mathrm{kV}, 5 \times 10^{-10}$ Amps probe current, $17 \mathrm{~mm}$ working distance, but also at $30 \mathrm{kV}$ with $26 \mathrm{~mm}$ WD. Standardisation of the BSE signal is achieved by use of brominated and iodinated dimethacrylate resin standards as previously described (Howell et al., 1998; Boyde et al., 1999). Samples were coated with carbon by evaporation. Whereas the thickness of the carbon coating is essentially irrelevant for the BSE SEM imaging case - since this element is transparent to electrons having energies in the range that we utilise - it is important for epi-fluorescence optical imaging since it acts as a neutral density filter in both excitation and emission. Generally, therefore, we prefer to perform any CSLM imaging in a first step. However, there are many instances where features of special interest are first revealed in the BSE mode, and satisfactory CSLM imaging can be achieved through the carbon coating layer.

\section{Development of novel software package}

The SEM that we use has a digital scan generator, which is a fundamental requirement for the correct stereologically based analysis of mineral concentrations in bone etc., derived from quantitative study using the BSE signal. In digital scan mode, we address a set of points to be analysed, and dwell at each point for long enough to reduce noise to an acceptable level. Imagine that we have two adjacent scanned points: these need not be 'pixels', since they may not and need not necessarily be adjacent points. One is black, the next white. With a conventional SEM scan generator, with the signal digitised 'on the fly', we will find a mid-grey value which is not there. We therefore decided that, having a digital scan generator, we would not interpolate between BSE data points. We leave the BSE image unchanged, and match the CSLM image to it, rather than the reverse. This we feel to be justified because the CSLM scan mechanism is not digital, though the signal is digitised. In CSLM, there is the very blurring between conceptual pixels (voxels) in the line which we have just mentioned. In addition, in most commercial instruments, the time dwelt on each pixel in a line is not equal.

Our first efforts in overlapping used Paint Shop Pro (version 5, Jasc Software Products Inc. Eden Prairie, MN 55344, USA http://www.jasc.com) which gives excellent opportunities to use basic modifications such as resizing (including XY magnification adjustments, i.e., changing pixel aspect ratios) and rotation. Each such step involves interpolation in the values assigned at each new pixel. If we consider the field taken by 40x lens by FCSLM as flat, these linear operations are sufficient. However, using Paint Shop Pro 5 is inconvenient if we have large sets of images to overlap. We therefore developed our own overlapping software package tailored to our needs, but there are probably others which already exist which would do the job, and ours may find applications in other areas. Our program uses a linear transformation matrix which projects one system to the other, which can be calculated by finding three corresponding points in the BSE and FCSLM pictures. This can be written as

$$
\vec{X}=\mathbf{A} \vec{X}^{\prime}+\vec{B}
$$

where $\vec{X}$ is the position of a point in the BSE image $\vec{X}^{\prime}$ is the position of the corresponding point in the FCSLM image, $\mathbf{A}$ is transformation matrix and $B$ is a translation vector of the system.

The elements of the matrix are

$$
\mathbf{A}=\left(\begin{array}{ll}
a & b \\
c & d
\end{array}\right)
$$


and of the translation vector are

$$
\vec{B}=\left(\begin{array}{l}
e \\
f
\end{array}\right)
$$

If we find three corresponding point pairs, which gives us six coordinates, than we can find the transformation matrix and translation vector by solving the equation for six unknowns:-

$$
\begin{aligned}
& x_{1}=a x_{1}^{\prime}+b y_{1}^{\prime}+e \\
& y_{1}=c x_{1}^{\prime}+d y_{1}^{\prime}+f \\
& x_{2}=a x_{2}^{\prime}+b y_{2}^{\prime}+e \\
& y_{2}=c x_{2}^{\prime}+d y_{2}^{\prime}+f \\
& x_{3}=a x_{3}^{\prime}+b y_{3}^{\prime}+e \\
& y_{3}=c x_{3}^{\prime}+d y_{3}^{\prime}+f
\end{aligned}
$$

In the next step, we use the equation $\vec{X}=\mathbf{A} \vec{X}^{\prime}+\vec{B}$ to transform the system of points from the FCSLM image, to the BSE system. If we use a pixel matrix of points $\vec{X}^{\prime}$ we get another pixel matrix $\vec{X}$. Where the pixels are not used we linearly interpolate between adjacent points. The centres of the coordinate systems are the central pixels in each image.

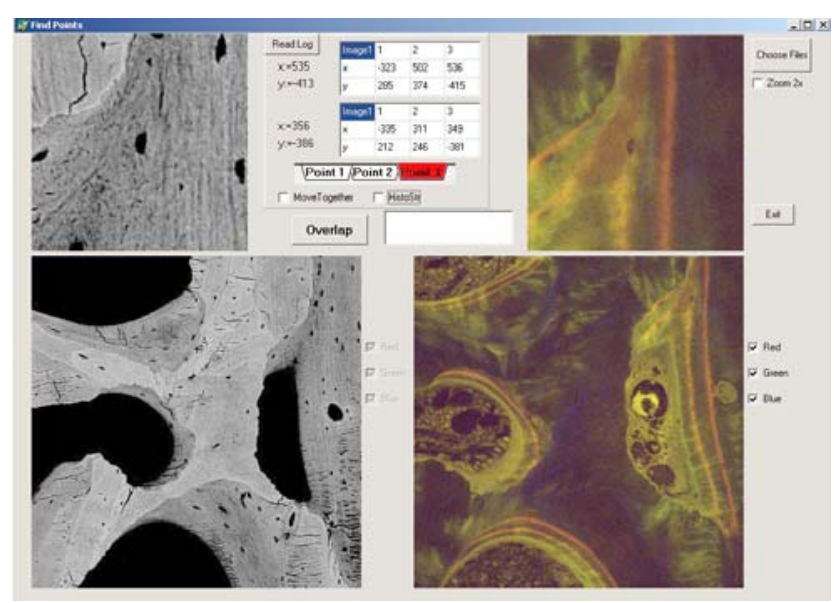

Figure 1. (Iliac crest biopsy from a 17 year old female who presented for surgical correction of a knee deformity secondary to calcium deficiency: Case no. 26 from the study of Schnitzler et al., 1994). Screen capture at step 1 , finding the corresponding BSE-SEM (left) and FCSLM (right) images: the yellow colour is due to tissue autofluorescence. Tetracycline double labelling (here shown as orange-red colours within bone domain) had been carried out using dimethyl chlortetracycline $300 \mathrm{mg}$ twice a day according to the schedule: 2 days labelling 10 days break -2 days labelling: the last dose at 8 days before biopsy. Field width of SEM image $=713 \mu \mathrm{m}$.

In practice, choosing the three points is certainly the main part of the whole problem. We have to make sure the points really correspond to each other. It is always best to choose the smallest features which can be seen straight away, rather than to choose the edge or the centre of a big feature because of misjudging the correspondence.

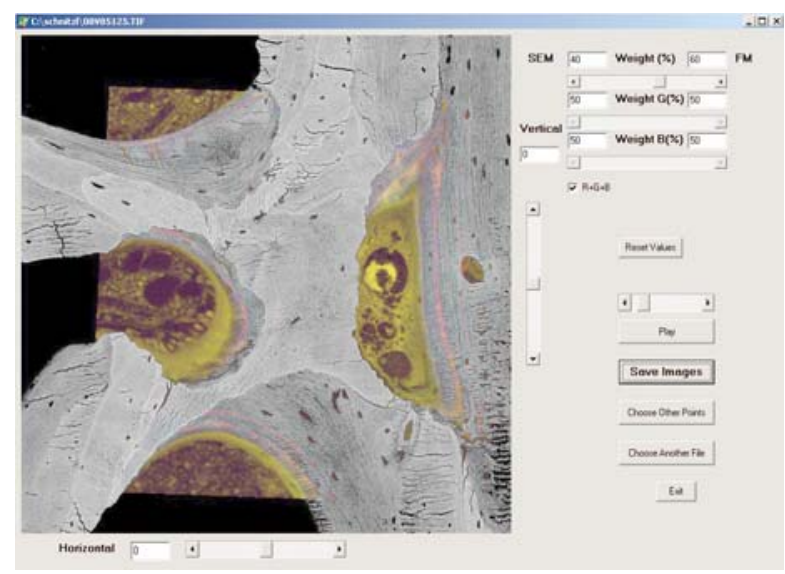

Figure 2. (Same as Fig.1.) Screen capture at step 4, having found three corresponding points in the BSESEM and fluorescence confocal images. The program allows the option to display the BSE and confocal components in the same screen space alternately, to check the choice of overlap. Here, the SEM image has been given $40 \%$ and the confocal $60 \%$ weight, allowing the tetracycline lines to be seen clearly in the bone domain.

There is a choice of ways in which to display the overlapped data. The BSE image is stored in 8 bits format (256 grey levels) and may have either a linear grey palette or a pseudo-colour look up table (LUT) to display mineralisation density class: further, it is often useful to apply a median filter operation to this image to simplify the classification of bone according to its degree of mineralisation. If we only record one channel of fluorescence colour in the FCSLM image, it is stored in 8 bits with a green palette. However, we may also have multiple fluorescence colours stored in RGB, 8 bits per channel, i.e. a 24 bit image. Further, we may have a stack of fluorescence mode images, and we may also have reflection mode confocal images. These cannot be combined in a single stationary image, but any combination can be combined as a movie image file, most commonly in AVI or GIF format.

Returning to the problem of producing a single overlapped image, we found the best way of overlapping is to change the BSE image to 24 bits, retaining the red and blue channels: in the green channel we may put either the pixel from the BSE or from the FCSLM image, whichever has the greater value. Note that bone marrow and osteocytic (cellular) space is essentially black and featureless in the BSE image, but is rich with relevant cellular detail in the FCSLM case. The BSE images are generally scaled in exactly the same way, the range of grey values reflecting the range of mineralisation levels found in bone (Boyde et al., 1999). - Levels in the FCSLM case vary from region to region and sample to 
sample. We may therefore also find it very interesting to vary the relative weights on the values from the BSE and the FCSLM images. (In practice, the value from the BSE image is multiplied by percentage of weight and the value from FCSLM image is multiplied by percentage to $100 \%$. Choosing $0 \%$ in the FCSLM image gives a resultant image which is the same as the original BSE image, and choosing $100 \%$ of the FCSLM image gives the BSE image, but with the green channel made by values from the FCSLM image. If we use $50 \%$, the BSE picture has the black area filled with the values from the FCSLM image and also has lines that correspond to intra-vital labelling and any other anomalously bright features within the bone. Giving more weight to FCSLM image fills the area of lower density of bone with a pink colour.

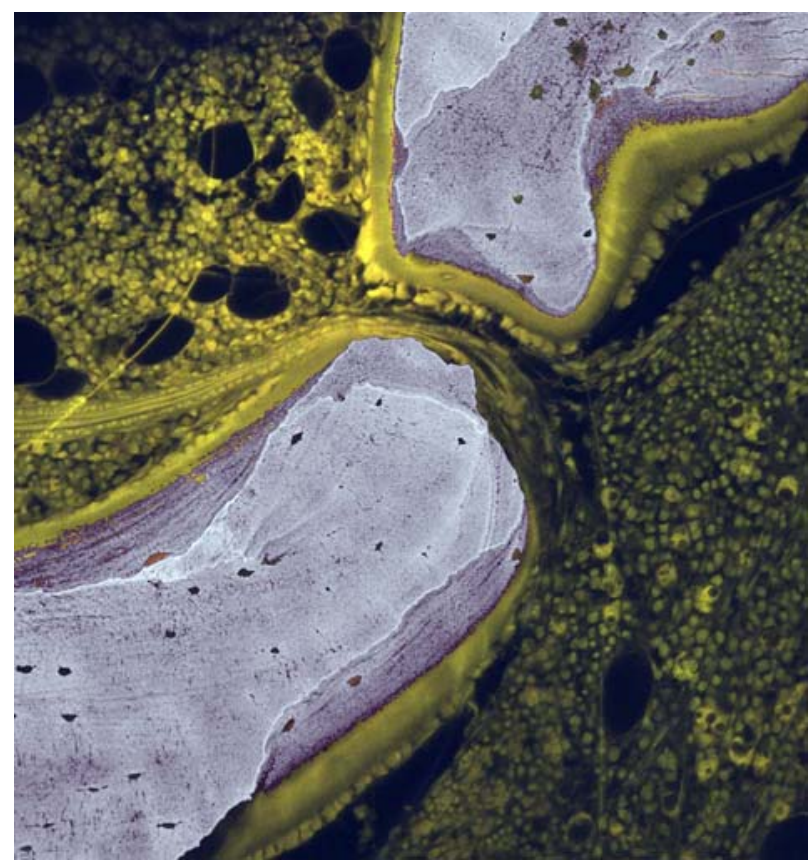

Figure 3. (Iliac crest biopsy from a 16 year old male teenager, case no.10 from the study of Schnitzler et al., 1994) Note substantial osteoid (non-mineralised bone matrix seams), and a small blood vessel. Field width $=560 \mu \mathrm{m}$.

A further development was to make the program suitable also for multiple fluorescence colour labelling. The program gives options to find corresponding points either displaying all three colour channels or any combination of the channels in the FCSLM image. Overlapping is done in a similar way as for the single colour labelling. The only difference is that each value from each channel is compared separately to the value of the BSE image. There is also another option which might be useful: the user can decide whether to put the same weight on each channel or to set the weight for each channel separately.

\section{Discussion}

The developments described here enable us to mix and match BSE SEM images of calcified tissues with those recorded using confocal microscopy. With a dry block surface imaged in air, the best correspondence will obviously be awaited with the confocal reflection mode image, particularly if this is a 'map' or a 'maximum intensity' [enhanced depth of field from through focussing image series] image (Boyde and Jones, 1995). If we use topographic mode BSE imaging - differencing the signal levels reaching opposing obliquely facing detector segments - then excellent correspondence with confocal reflection imaging is obtained. This may be useful in matching fields from BSE and CSLM, but neither mode is essentially useful in studying biological problems relating to bone. Here, the useful data derive from compositional BSE and fluorescence confocal imaging. One further method may be of value, namely block surface staining for conventional LM imaging either by trans-illumination or by making the block self luminous via its general autofluorescence. The procedures for field matching will, however, be the same.

The information depth in 20kV BSE SEM imaging of bone is of the order of one micron, with most signal deriving within the top one half micron (Howell and Boyde, 1999). Much has been written about 'optical slice thickness' in CSLM, and most of this is relevant here. Briefly, signal intensity is greatest from the layer within which the exciting beam is narrowest. In the usual case, an operator might regard the top surface of the sample to be in focus when the first, brightest fluorescence image is obtained. This criterion will result in the focus being depending on the numerical aperture (NA) of the objective and the size of the confocal pinhole - some microns deep to the real surface. In this case one can await poorer correspondence between features imaged in the SEM and CSLM. The best criterion for best focus is to use the reflected light mode to find the surface. This, however, may result in the focus being marginally too superficial, since it is exactly what is just below the surface that we need to represent if CSLM is to be matched really well to BSE data. Obviously the use of lower NA objectives must result in a poorer discrimination of the surface from subsurface layers,

The surface of the block must not only be flat, but it must not be tilted. With larger PMMA blocks - in which we ensure that the front surface is parallel to the back from the way in which the sample is clamped during the milling process - then this is not so much of a problem. However, confocal microscope manufacturers should pay more care to the manner in which it is arranged that a plane parallel object is held perpendicular to the optic axis. If the sample has been polished, it is much more difficult to arrange that the top surface of the sample is normal to the optic axis, and, of course, polished samples have local variations from flatness.

Another problem arises from deficiencies in the objective lenses per se. Until dry lenses are used with mirror surfaces in a CSLM in reflection mode, one will 
never appreciate the magnitude of this difficulty. It will not usually be noticed with typical biological samples examined with immersion lenses focussed away from the coverslip. Little is written about this non-flat-field problem. We pay heavily for the assignation 'PLAN' (aplanatic) in the description of an objective lens, but they are never flat enough. For a perfect match of BSE and FCSLM, we would want the optical beam to be focussed at the surface. If it is focussed at the centre of the field, it is generally focussed just above the surface at the field peripheries, an effect easily seen, for example, with the 10/0.40 objective used in the present studies. This is generally not observed in biological practice because typical samples are several microns thick, and a stack of images may be recorded. Particularly when we are working with tissue autofluorescence (AF), we may want to minimise photobleaching by recording only the one field depth which matches best with the BSE data layer, but it is also of interest to study the changes in tissue properties from the block surface downwards in order to have a better 3D perspective, with added temporal information from any dynamic, intra-vital, tissue-formation labels.

\section{Conclusion}

A method is presented for the cross-comparison of backscattered electron imaging in an SEM and confocal fluorescence imaging of the same layer in the surface of a flat block of tissue. It is illustrated with examples of bone tissue with abundant osteoid, to demonstrate the value of filling in the otherwise empty, dark space in the BSE image. It will, however, be of value in many bone studies, including those employing fluorochrome labels to study temporal aspects of bone formation, and in skeletal and 'dental' implant applications.

\section{Ackowledgements}

Ludek Lovicar and Jan Zamecnik were Erasmus/Socrates exchange students. We thank the Horserace Betting Levy Board for financial support and Roy Radcliffe and Mo Arora for technical assistance. The SEM facility for the determination of bone mineralisation density at the microscopic scale was funded by the MRC.

\section{References}

Boyde A (1987) Applications of Tandem scanning Reflected Light microscopy and 3-dimensional imaging. Ann New York Acad Sci 483: 428-439.

Boyde A, Jones SJ (1983) Backscattered electron imaging of skeletal tissues. Metab Bone Dis Rel Res 5:145150 .

Boyde A, Jones SJ (1995) Mapping and measuring surfaces using reflection confocal microscopy. In: Handbook of Biological Confocal Microscopy, 2nd edition, pp255-266: JB Pawley (Ed) Plenum Press.
Boyde A, Reid SA (1983) Tetracycline cathodoluminescence in bone, dentine and enamel. Histochem 77: 525-533.

Boyde A, Reid SA (1984) New biological applications for the CL imaging mode in the scanning electron microscope. In: Electron Microscopy 3: Life Sciences II, Csanady A, Rohlich P, Szabo D (Eds.), Budapest, Hungary. pp 1813-1814

Boyde A, Wolfe LA (2000) Spatial and Temporal Patterns of Bone Growth around Endosseous Implants: A Critical Study of Microscopic Methods. Chapter 29 in "Bone Engineering", J.E. Davies (Ed.), em squared Inc., Toronto, Canada. pp 321-331.

Boyde A, Jones SJ, Taylor L, Wolfe L, Watson TF (1990) Fluorescence in the tandem scanning microscope. J Microsc 157: 39-49.

Boyde A, Wolfe L, Maly M, Jones SJ (1995) Vital confocal microscopy in bone. Scanning 17: 72-85.

Boyde A, Travers R, Glorieux FH, Jones SJ (1999) The mineralisation density of iliac crest bone from children with osteogenesis imperfecta. Calcif Tiss Int 64: 185-190.

Frost HM (1962) Tetracycline labelling of bone and the zone of demarcation of osteoid seams. Can J Biochem Physiol. 40: 485-489.

Goldman HM, Blayvas A, Boyde A, Howell PGT, Clement JG, Bromage TG (2000) Correlative Light and Backscattered Electron Microscopy of Human Bone. Part II: Automated Image Analysis. Scanning 22: 337-344.

Howell PGT, Boyde A (1999) Surface roughness of preparations for backscattered electron scanning electron microscopy: the image differences and their Monte Carlo simulation. Scanning 21: 361-367.

Howell PGT, Davy KWM, Boyde A (1998) Mean atomic number and backscattered electron coefficient calculations for some materials with low mean atomic number. Scanning 20: 35-40.

Hulth A, Olerud S (1962) Tetracycline labelling of growing bone. Acta Soc Med Upssala 67: 219-231.

Rahn BA, Perren SM (1970) Calcein blue as a fluorescent label in bone. Experientia. 26: 519-520.

Rahn BA, Perren SM (1971) Xylenol orange, a fluorochrome useful in polychrome sequential labeling of calcifying tissues. Stain Technol. 46: 125-129.

Roschger P, Plenk H Jr, Klaushofer K, Eschberger J (1995) A new scanning electron microscopy approach to the quantification of bone mineral distribution: backscattered electron image grey-levels correlated to calcium Kalpha-line intensities. Scanning Microscopy 9: 75-88.

Schnitzler CM, Pettifor JM, Patel D, Mesquita JM, Moodley GP, Zachen D (1994) Metabolic bone disease in black teenagers with genu valgum or varum without radiologic rickets: a bone histomorphometric study. J Bone Miner Res. 9: 479-486.

Vajda EG, Skedros JG, Bloebaum RD (1998) Errors in quantitative backscattered electron analysis of bone standardized by energy-dispersive x-ray spectrometry. Scanning 20: 527-535. 


\section{Discussion with Reviewers}

H Plenk: Is the software available, and how much experience would be needed?

Authors: Anyone with digital SEM and confocal can duplicate this work. I hope to encourage others to do so. Individuals could, and no doubt will, contact us for software, but there must be many registration programmes out there which will function just as well.

H Plenk: Technique: The photobleaching effect in FCSLM was apparently a point of concern, but is not also the electron beam damage to the scanned area of the block surface in SEM a point of concern, if BSE SEM precedes FCSLM?

Authors: We note that BSE preceded CSLM, and this is one of the reasons. BSE $>$ CSLM is of concern if the beam loading is high, as in high magnification images.
H Plenk: This paper discusses only the surely important technical aspects of surface flatness etc., but not possible advantages or disadvantages of this technique when investigating bone-implant interfaces. For example, how would reflections of a polished or micro-milled metallic surface influence the CSLM images, or would a light or electron beam scattering at the metal-tissue interface interfere with the clarity of the images?

Authors: The intense reflection from metal surfaces will always be well represented even in the least well designed commercial CSLMs, and this will help to determine the ideal plane of focus. However, many have a 'hot spot' in reflection mode due to unwanted reflections from the near centre of lens surfaces. 